American Journal of Infectious Diseases 3 (3): 142-150, 2007

ISSN 1553-6203

(C) 2007 Science Publications

\title{
Cholesterol Metabolism in Brain and Skin Fibroblasts from Sarda Breed Sheep With Scrapie-resistant and Scrapie-susceptible Genotypes
}

\author{
${ }^{1}$ Alessandra Pani, ${ }^{2}$ Claudia Abete, ${ }^{2}$ Claudia Norfo, ${ }^{2}$ Claudia Mulas, ${ }^{2}$ Marirosa Putzolu, ${ }^{2}$ Sergio Laconi, \\ ${ }^{1}$ M.Dolores Cannas, ${ }^{1}$ Cristina D. Orrù, ${ }^{1}$ Paolo La Colla and ${ }^{2}$ Sandra Dessi \\ ${ }^{1}$ General Microbiology and Virology and Microbial Biotechnologies Section, \\ ${ }^{2}$ Experimental Pathology Section, Department of Biomedical Sciences and Technology, \\ University of Cagliari, Italy
}

\begin{abstract}
Scrapie is a fatal spongiform encephalopathy of sheep, a transmissible form of prion disease caused by neuronal accumulation of the aberrantly conformed prion protein $(\mathrm{PrPsc})$. Currently, no ante-mortem diagnostic tests are available to detect this untreatable disease in the pre-clinical stage, thus making difficult to control its spread. Recent evidence suggests that the production of PrPsc can be modulated by the levels of membrane cholesterol in neuronal cells. Since cholesterol levels in cell membranes are dependent on cholesterol homeostasis in the whole organism, we studied cholesterol metabolism in brain tissues, plasma and skin fibroblasts of Sarda breed sheep with scrapie-resistant (ARR/ARR) and scrapie-susceptible (ARQ/ARQ) prion protein genotypes, both not infected (ARQ/ARQ-) and infected (ARQ/ARQ+) with scrapie. We found that, the levels of cytoplasmic cholesterol esters (CE) in brains and skin fibroblasts from sheep with the ARQ/ARQ genotype were consistently higher than those from sheep with the ARR/ARR genotype. Conversely, the levels of free cholesterol (FC) were lower in ARQ/ARQ, as compared to ARR/ARR sheep, thus resulting in a sharp reduction of the $\mathrm{FC} / \mathrm{CE}$ ratio. Moreover, both uninfected and infected ARQ/ARQ sheep showed abnormally low levels of high density lipoprotein-cholesterol (HDL-C) in their plasma, as compared to ARR/ARR sheep. These data other than adding new strength to the notion that altered levels of intracellular cholesterol may indicate the presence of a lipid metabolic state that predisposes to infection with, and accumulation of, PrPsc in the brain, discriminate for the first time between two distinct but related cellular pools of cholesterol, namely membrane FC on one hand and cytoplasmic $\mathrm{CE}$ on the other.
\end{abstract}

Key words: Scrapie, Prion diseases, Cholesterol homeostasis, Cholesterol levels, Rafts

\section{INTRODUCTION}

Prion diseases are fatal neurodegenerative disorders for which no diagnostic tests "in vitam", nor treatments are currently available. Unlike any other neurodegenerative disease, prion diseases can be inherited, transmitted or sporadic. However, in spite of their diverse origin, all these forms are caused by the conversion of the normal prion protein $(\mathrm{PrPc})$ into its pathologic, protease-resistant counterpart $(\mathrm{PrPsc})^{[1]}$. The difference between $\operatorname{PrPc}$ and PrPsc is conformational: $\mathrm{PrPc}$ is predominantly alpha helical and contains few beta-sheets, while PrPsc is mainly composed of beta-sheets ${ }^{[2]}$. Under normal conditions, the majority of $\mathrm{PrPc}$ is catabolized by cleavage at residues 110 and 111 , and only a limited amount of it eventually switches to PrPsc ${ }^{[3]}$. By contrast, in prion diseases the conversion rate of $\mathrm{PrPc}$ to PrPsc is greatly enhanced ${ }^{[4]}$ leading to accumulation of cytotoxic, protease-resistant PrPsc aggregates, the key event in the initiation of the neuropathological manifestations of the diseas $^{[4]}$. Interestingly, intracerebral injection of exogenous PrPsc in mice induces the development of encephalopathy only in animals that express the endogenous PrPc, but not in PrPc-null mice, indicating that expression of the endogenous PrPc protein is an essential condition for the development of prion diseases ${ }^{[5]}$.

$\operatorname{PrPc}$ is normally anchored to specialized cholesterol-rich domains of the plasma membrane, termed lipid rafts or caveolae, through its glycophospatidyl inositol (GPI)-terminus ${ }^{[6]}$. These membrane domains are detergent-insoluble regions

Corresponding Author: $\quad$ Sandra Dessi, Università di Cagliari, Dipartimento di Scienze e Tecnologie Biomediche,Sezione di Patologia Sperimentale Via Porcell 409124 Cagliari, Italy T. n 00390706758340 Fax n 0039070662574 
characterized by the presence of free cholesterol (FC), saturated phospholipids and raft-resident proteins ${ }^{[7,8]}$. Increasing evidence indicates that subtle intracellular cholesterol changes affect the intracellular processing/trafficking, function and/or activation of raft-resident proteins ${ }^{[9,10]}$. Therefore, a role for cholesterol in the metabolism of PrPc and its conversion to PrPSc has been proposed ${ }^{[11,12]}$.

In normal tissues, with the exception of liver and adrenal glands, approximately $90 \%$ of the total cellular cholesterol resides in membrane raft domains as FC, while only a minor amount (approximately $1-10 \%$ ) is found as cholesterol esters (CE) in a cytoplasmic storage form ${ }^{[13]}$. Since FC levels are critical to the maintenance of proper membrane fluidity, as well as to the function and/or activation of raft-resident proteins, cells have developed a highly integrated set of homeostatic mechanisms acting in concert to finely control intracellular cholesterol levels ${ }^{[14]}$.

The FC found in cell membranes derives either from de novo synthesis in the endoplasmic reticulum (ER), or from lysosomes following receptor-mediated uptake of low-density lipoproteins (LDLs). Membrane $\mathrm{FC}$, however, is in a dynamic state, moving back to the ER in response to changing homeostatic conditions in the cell ${ }^{[15]}$. In fact, if cholesterol in the rafts exceeds threshold levels, its excess is promptly transferred to the ER, where it might elicit high-order responses from the control proteins embedded ${ }^{[16]}$. FC in the ER blocks the function of its cellular sensor, the SREBP-cleavageactivating-protein (SCAP), which is needed for the proteolytic activation of the sterol-regulatory-elementbinding-protein-2 (SREBP-2), a transcriptional factor that promotes the expression of genes involved in cholesterol synthesis [i.e. hydroxyl-methyl-glutarylcoenzime A-reductase (HMGCoA-R)] and uptake (i.e. LDL receptor) ${ }^{[15-18]}$. FC in the ER can be either used in the assembly of newly formed membrane rafts, and returned to the plasma membrane through vesicular transport, or, if in excess, converted to CE by the acylcholesterol-acyl-transferase (ACAT) and stored in the cytoplasm as neutral lipid droplets ${ }^{[19]}$. Viceversa, when cell membranes need FC, or when CE droplets exceed a critical threshold value, $\mathrm{CE}$ can be reconverted to $\mathrm{FC}$ by the neutral-cholesterol-ester-hydrolase $(\mathrm{nCEH})^{[20]}$. FC can then be recycled to the membranes by cholesterol binding protein, such as caveolin-1 (Cav-1) ${ }^{[7,8]}$, where i) it can be used to replenish raft domains, or if in excess, ii) delivered to extracellular acceptors, namely high-density lipoproteins (HDL), via the ATP-bindingcassette-sub-family A-member 1 (ABCA-1) transporter (21).

Recent evidence indicates that the metabolic fate of $\mathrm{PrPc}$ is dependent on its location in the plasma membrane which, in turn, is affected by cholesterol levels in the rafts ${ }^{[22]}$. On the other hand, cholesterol levels in the rafts can be affected by various factors that may alter its intracellular metabolism, including the levels of Cav-1 transporter ${ }^{[23]}$. These findings led us and others ${ }^{[24,25]}$ to hypothesize that cholesterol may have a pivotal role in the PrPc to PrPsc conversion and, therefore, that alterations in intracellular cholesterol homeostasis may predispose to prion diseases.

Scrapie is the ovine counterpart of prion disorders, such as Creutzfeld-Jacobs in humans and BSE in cows [26-29]. Although the transmissible origin of sheep scrapie is well documented, there is also evidence for a genetic susceptibility that depends on the particular polymorphisms of the prion protein gene $(P R N P)^{[26-29]}$.

Many polymorphic codons have been described to date in the PrPc open-reading frame ${ }^{[30]}$. However, only substitutions at codons $136(\mathrm{~A} \rightarrow \mathrm{V}), 154(\mathrm{R} \rightarrow \mathrm{H})$ and $171 \quad(\mathrm{Q} \rightarrow \mathrm{R} \quad$ or $\mathrm{H})$ are associated with susceptibility/resistance to the disease. These substitutions generate different alleles: VRQ, ARQ, AHQ, ARH, ARR and 15 different genotypes. In general, VRQ and ARQ alleles are associated with high susceptibility to scrapie, while the ARR allele is associated with resistance ${ }^{[30]}$.

In the present study we compared the cholesterol metabolism of Sarda breed sheep carrying the scrapieresistant genotype (ARR/ARR) with that of animals carrying the scrapie-susceptible (ARQ/ARQ) genotype, both without (ARQ/ARQ-) and with (ARQ/ARQ $\left.{ }^{+}\right)$ scrapie. The aim of the present study was to establish a possible correlation between cholesterol homeostasis and genetic susceptibility to scrapie, in an attempt to identify a lipid metabolic state that may influence genetic predisposition to prion diseases.

\section{MATERIALS AND METHODS}

Brain samples: Brain samples collected from 3 adult sheep from the experimental farms of the Istituto Zooprofilattico Sperimentale of Sardinia, Italy, were a generous gift by Dr. Ciriaco Ligios. Two sheep carried 
the susceptible scrapie genotype ARQ/ARQ: 1 sheep was clinically affected by natural scrapie while the other was healthy and negative for $\operatorname{PrP}^{\mathrm{Sc}}$ in the brain, by Western blot analysis. The third sheep carried the resistant genotype ARR/ARR. All sheep were euthanized with a barbiturate followed by $4 \mathrm{ml}$ of embutramide and mebenzonico-iodide (Hoechst Roussel Vet), brain samples were stored at $-80^{\circ} \mathrm{C}$ immediately after collection and thawed just prior to use.

Skin biopsies and fibroblast isolation: Fresh skin biopsies (a generous gift by Dr. Ciriaco Ligios) obtained from 14 sheep at the experimental farms of the Istituto Zooprofilattico Sperimentale of Sardinia, Italy, were delivered to our laboratory in transport medium (Eurocollins). All animals were raised in the same environmental conditions and were of the same age and sex in order to reduce physiological differences of biochemical and molecular determinations. Ten biopsies were from sheep with the susceptible genotype ARQ/ARQ: of these, 2 were scrapie-free, 1 had natural scrapie, while 7 developed clinical disease following experimental inoculation of scrapie. All biopsies were taken from scrapie-affected sheep at the terminal clinical stage of the disease. In addition, skin biopsies from 4 scrapie-resistant ARR/ARR sheep which were experimentally inoculated with PrPsc and remained free of clinical signs were used as controls.

Tissues fragments from skin biopsies were plated into 6-well plates and allowed to adhere to the bottom of the vessels. Then, after 2 hrs, they were covered with a few drops of Dulbecco's modified Eagle's medium (D-MEM) (Gibco Lab NY, USA) supplemented with $10 \%$ fetal bovine serum (FBS) (Sigma), $100 \mathrm{U} / \mathrm{ml}$ penicillin/streptomycin (Sigma), and fungizone (Life Technologies, Inc.) and incubated at $37^{\circ} \mathrm{C}$ in a humidified incubator with $5 \% \mathrm{CO}_{2}$. The following day, tissue fragments were overlaid with culture medium, which was changed every two days. Five to six days later, fibroblasts begun to proliferate from the fragment margins ("halo of cells") and to form a monolayer. After four weeks, fibroblasts were purified by repeated trypsinization (trypsin-EDTA) and passaging to achieve an homogenous population of spindle cells. Purified fibroblasts were washed twice with sterile PBS and centrifuged. $1 \times 10^{6}$ cells were then seeded into $25 \mathrm{~cm}^{2}$ culture flask and grown to confluence. Then, cells were either used for "in vitro" experiments or resuspended in cryopreservation medium at a density of $1 \times 10^{7}$ cells $/ \mathrm{ml}$ and stored in liquid nitrogen. Analytical assays were carried out using fibroblast cultures between the second and fourth passage. Cells were plated at a density of $5 \times 10^{3} \mathrm{cell} / \mathrm{cm}^{2}$ in 6 -well plates and brought to proliferative quiescence by incubation for $48 \mathrm{hrs}$ in serum depleted ( $0.2 \%$ FCS) MEM 199. Then, quiescent cells were stimulated to re-enter the cell cycle by the addition of $10 \% \mathrm{FCS}$ and incubation at $37^{\circ} \mathrm{C}$ for the required time intervals.

Blood sampling: From the same group of sheep that provided skin biopsies, blood samples were also obtained and used for determination of plasma total cholesterol (TC) and high-density lipoprotein cholesterol (HDL-C) by an enzymatic method (Carlo Erba, Milan Italy).

\section{Brain lipid extraction and cholesterol} determination: Minced tissue samples were homogenized and neutral lipids were extracted ${ }^{[31]}$. FC and $\mathrm{CE}$ were separated by thin layer chromatography and their respective levels were determined by the method of Bowman and Wolf ${ }^{[32]}$ using cholesterol and cholesterol oleate as standards.

Neutral lipid staining: The neutral lipid content of cultured fibroblasts was determined as follows. Cultures were washed three times with PBS and fixed by soaking in $10 \%$ formalin. Cells were then treated with isopropyl alcohol $(60 \%)$, washed, stained with oil red $O$ (a stain specific for intracellular neutral lipid droplets) and counterstained with Mayer's hematoxylin. Stained cells were examined by light microscopy and digital images were recorded. Red color intensity in single cells, indicating neutral lipid-bound oil red $\mathrm{O}$, was measured by the NIH Image 1.63 Analysis Software program (Scion Image). Values were expressed as the mean colour intensity calculated on at least 30 single cells in 6 different microscopic fields.

${ }^{3} \mathrm{H}$-Thymidine incorporation: Cell proliferation rates were measured by ${ }^{3} \mathrm{H}$-thymidine incorporation. Skin fibroblasts were cultured as described. Cells were labeled with $2.5 \mu \mathrm{Ci} / \mathrm{ml}$ of ${ }^{3} \mathrm{H}$-thymidine (Amersham, 5 
$\mathrm{mCi} / \mathrm{mmole}$ ) during the last $6 \mathrm{hrs}$ before sample collection and harvested at the time points indicated. Cells were rinsed twice with ice-cold PBS, washed with $5 \%$ cold TCA, repeatedly washed with water and then lysed with $1 \mathrm{M} \mathrm{NaOH}$ at room temperature. The amount of acid-insoluble radioactivity was determined in a Beckman $\beta$ counter (Palo Alto, Ca) using Ultima Gold as the scintillation fluid. Aliquots of cell lysates were processed for protein content determination by the Lowry method ${ }^{[33] .}$ Cell viability was assessed by the trypan blue exclusion test.

Cholesterol esterification: Cholesterol esterification was evaluated by incubating fibroblast monolayers for $6 \mathrm{hrs}$ in medium containing $5 \mu \mathrm{Ci} / \mathrm{ml}$ of $\left[1-{ }^{14} \mathrm{C}\right]$ oleic acid (Dupont, NEN $55 \mathrm{mCi} / \mathrm{mmol}$ ) bovine serum albumin (BSA) complex. After incubation, cells were washed with PBS, to remove the bulk of radioactive precursor, and lipids were extracted with acetone. Lipid subclasses were separated by thin layer chromatography (TLC) on kiesegel plates using a solvent system containing n-heptane/isopropyl ether/formic acid $(60: 40: 2 \mathrm{v} / \mathrm{v} / \mathrm{v})$. Cholesterol ester bands were identified by comparison with reference standard run simultaneously side-by-side and visualized under iodine vapors. To determine the amount of lipids incorporating $\left[1-{ }^{14} \mathrm{C}\right]$ oleate-cholesterol, bands were excised and added directly to counting vials containing $10 \mathrm{ml}$ of Econofluor (DuPont NEN) liquid scintillation fluid.

Statistical analysis. Data represent mean values \pm standard error (SE). Statistical comparisons were performed with the Student $t$ test. Significance was set at $\mathrm{P}<0.05$.

\section{RESULTS}

Cholesterol profiles in sheep brains and plasmas: We first determined cholesterol levels in the brains from sheep carrying the scrapie-resistant genotype (ARR/ARR), or from animals carrying the scrapiesusceptible genotype, either not infected (ARQ/ARQ') or infected (ARQ/ARQ ${ }^{+}$) with scrapie (Fig. 1). Panel A shows that TC and FC levels were significantly higher $(\mathrm{P}<0.05)$ in brain samples from the scrapie-resistant sheep as compared to scrapie-susceptible animals, irrmannt:vely to whether they were unaffected (A C $2 Q^{-}$) or affected (ARQ/ARQ ${ }^{+}$) by scrapie. Conversely, CE levels were 1.5-fold lower in $\mathrm{ARR} / \mathrm{ARR}$ sheep, as compared to both $\mathrm{ARQ} / \mathrm{ARQ}^{-}$and $\mathrm{ARQ} / \mathrm{ARQ}^{+}$animals. The above differences in cholesterol levels are unequivocally evident by considering the CE:TC ratio which, in brain samples from ARR/ARR sheep, is two-fold lower than in ARQ/ARQ counterparts.

On the other hand, plasma TC levels turned out to be comparable in scrapie-resistant and scrapiesusceptible sheep $(\mathrm{P}<0.05)$, whereas plasma HDL-C levels and, consequently, their percentage with respect to $\mathrm{TC}$, were found to progressively decrease in the following order: ARR/ARR > ARQ/ARQ ${ }^{-}>$ $A R Q / A^{+} Q^{+}$. In summary, when compared to sheep with the ARR/ARR genotype, ARQ/ARQ animals have, in their brains, lower absolute TC levels, lower $\mathrm{FC}$ and higher CE levels, and, in their plasma, lower HDL-C levels.

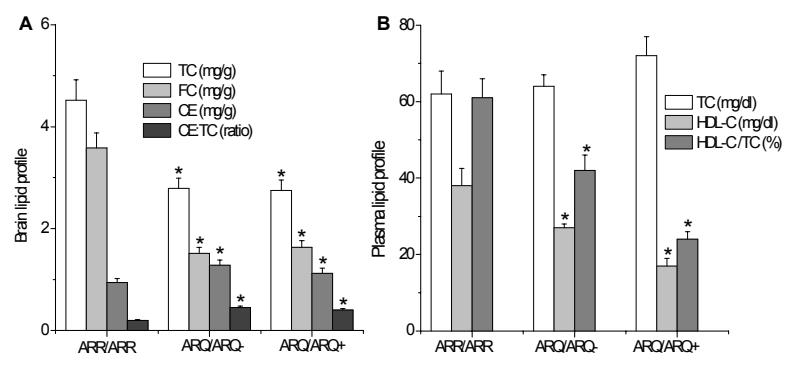

Fig 1: Cholesterol profiles in brain samples (A) and plasma (B) of sheep carryng a scrapie- resistant (ARR/ARR) or a scrapie-susceptible genotype either not infected (ARQ/ARQ) with scrapie. Lipid extraction and cholesterol determination are reported in Material and Method. Brain data represent mean values $\pm \mathrm{SE}$ of triplicate determinations of four samples from each brain. Plasma data represent mean value \pm SE of triplicate determinations of samples from 4 ARR/ARR, 2 ARQ/ARQ and 8 ARQ/ARQ sheep. ${ }^{*} \mathrm{P}<0.05$ vs ARR/ARR

Altogheter our results support the conclusion that high brain CE levels (which may be considered an index of altered cholesterol homeostasis), coupled with low HDL-C in plasma, may represent a distinctive metabolic profile of scrapie-susceptible animals.

\section{Cholesterol metabolism in quiescent and replicating} sheep skin fibroblasts: The metabolic and biochemical alterations described in the brain of patients affected by various neurodegenerative diseases, such as the Alzheimer Disease, have been also found in peripheral tissues ${ }^{[34]}$.

Therefore, we investigated whether the same cholesterol metabolic profile found in brains of scrapie- 
resistant and -susceptible sheep could also be found in skin fibroblasts. In this case, the levels of cytoplasmic CE-containing neutral lipids (Fig. 2) were determined in both quiescent and proliferating fibroblasts isolated from skin biopsies of ARR/ARR, ARQ/ARQ ${ }^{-}$and $\mathrm{ARQ} \mathrm{ARQ}^{+}$sheep (for details, see Materials and Methods). Visual inspection of the various samples reveals that, immediately after stimulation $(0 \mathrm{hr})$, stained neutral lipid droplets were less clearly evident in skin fibroblasts from ARR/ARR sheep than in cells from both $\mathrm{ARQ} / \mathrm{ARQ}^{-}$and $\mathrm{ARQ} / \mathrm{ARQ}^{+}$animals. Concomitantly with beginning of cell proliferation, CEcontaining lipids droplets appear to increase in all cultures, although they remain significantly higher in ARQ/ARQ fibroblasts (data not shown). In order to obtain a reliable quantification of CE-containing lipids, the stain intensity/cell was measured as detailed in Materials and Methods. The results of this quantitative analysis, reported in Fig. 2, confirm what suggested by the visual inspection of cell cultures, namely, the levels of CE-containing lipids/cell were always higher in ARQ/ARQ as compared to ARR/ARR cells, in both quiescent and proliferating cultures.

Next, the rate of CE synthesis in quiescent and proliferating fibroblasts from scrapie-resistant and susceptible sheep was determined by measuring the amount of ${ }^{14} \mathrm{C}$-CE synthesized during labelling with the ${ }^{14} \mathrm{C}$-oleate precursor.

As shown in Fig. 3A, shortly (6 hrs) after growth stimulation, the rate of ${ }^{14} \mathrm{C}-\mathrm{CE}$ synthesis was far lower in scrapie-resistant fibroblasts than in scrapiesusceptible cells. $24 \mathrm{hrs}$ after stimulation, the rate of ${ }^{14} \mathrm{C}-\mathrm{CE}$ synthesis reached the lowest level obtained in all cultures, irrespective of their genotype. The rate of synthesis remained around those low levels for up to 72 hrs after stimulation in ARR/ARR fibroblasts, while in ARQ/ARQ ${ }^{-}$fibroblasts it showed a modest increase (1.4-fold) during the same time frame. ${ }^{14} \mathrm{C}$-CE levels showed a more prominent increase (3-fold and 3.5-fold at 48 and $72 \mathrm{hrs}$., respectively) in ARQ/ARQ ${ }^{+}$ fibroblasts.

As revealed by ${ }^{3} \mathrm{H}$-thymidine incorporation (Fig. 3B), shortly (6 hrs) after growth stimulation the rate of DNA synthesis was, as expected, very low, irrespective of the fibroblast genotypes. At later times after growth stimulation, the rate of ${ }^{3} \mathrm{H}$-thymidine incorporation showed a steady but modest increase in ARR/ARR, reaching its peak (8-fold compared to time 6) at 48 hours. Conversely, the rate of DNA synthesis rose sharply after stimulation in ARQ/ARQ fibroblasts, reaching its peak (25-30-fold compared to time 6) at 24 hours and declining thereafter.

In conclusion, important differences were observed between ARR/ARR and ARQ/ARQ cultured skin fibroblasts in the rates of $\mathrm{CE}$ and DNA synthesis. First of all, the metabolic pathway leading to CE synthesis in quiescent fibroblasts was much more active in scrapiesusceptible than in scrapie-resistant fibroblasts.

Consistently with previous observations in other cell systems [35,36], increased rates of cholesterol esterification were associated with increased growth rates, mainly in skin fibroblasts from scrapiesusceptible sheep. These results indicate that skin fibroblasts from ARQ/ARQ sheep display the same set of metabolic changes observed in brain tissue from the same animals. Thus, cultured skin fibroblast may offer the advantage of allowing to avoid the frequent artefacts due to rapid post-mortem alterations of brain tissue and, being easily accessible, they could represent good candidates for the identification of new surrogate markers for ante-mortem diagnosis of prion diseases.

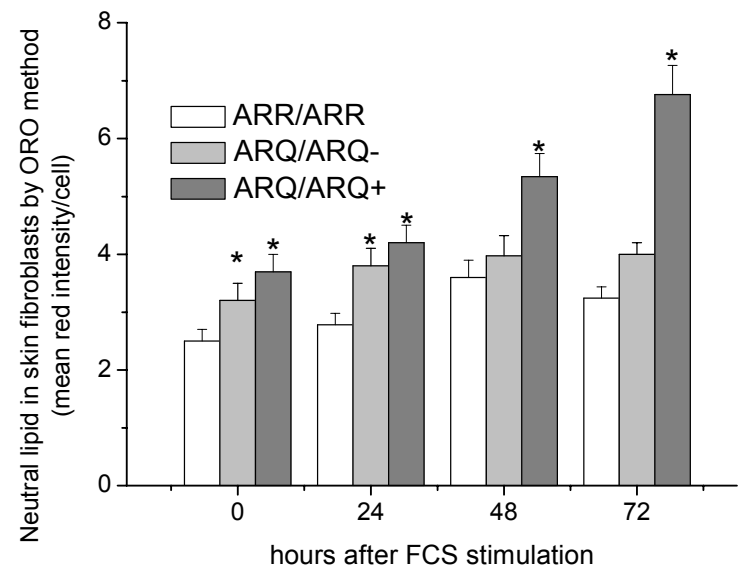

Fig 2: Oil-red O-stained neutral lipids of cultured skin fibroblasts from sheep carrying scrapie-resistant or -susceptible genotypes. Skin fibroblasts were cultured for 2 days in serum-depleted MEM-199 to induce a quiescent state. Then, their proliferation was stimulated by the addition of $10 \%$ FCS and samples were harvested 0, 24, 48 and $72 \mathrm{hrs}$ later. Figure reports the neutral lipidbound oil red $\mathrm{O}$, quantified as indicated in Materials and Methods, present in cells at various times after growth stimulation. Bars represent mean values \pm SE of at least 30 cells in 5 different microscopic fields from 4 ARR/ARR sheep, 2 ARQ/ARQ- sheep and 8 ARQ/ARQ+ sheep. $* \mathrm{P}<0.05$ vs ARR/ARR sheep 


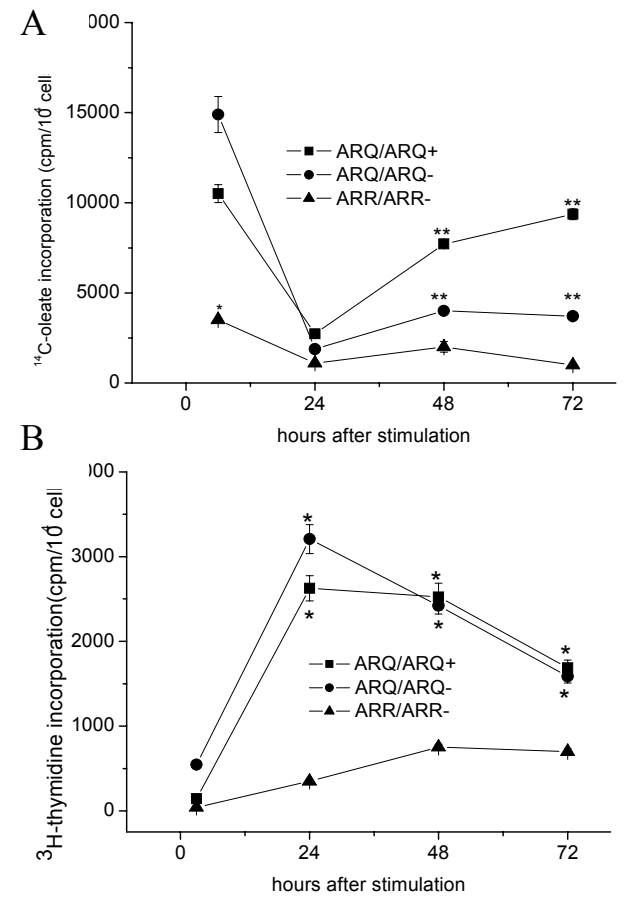

Fig 3: ${ }^{14} \mathrm{C}$-oleate (A) and ${ }^{3} \mathrm{H}$-thymidine (B) incorporation in cultured skin fibroblasts from sheep carrying scrapie-resistant or -susceptible genotypes. Radioactive precursors were added 6 hrs before harvesting and processing for determination of incorporated radioactive precursors. The amount of radioactivity was measured 6, 24, 48 and $72 \mathrm{hrs}$ after growth stimulation. Data represent mean values \pm SE of skin fibroblast samples from 4 ARR/ARR, 2 ARQ/ARQ- and 8 ARQ/ARQ+ sheep.

$* \mathrm{p}<0.05$ vs ARR/ARR sheep.

\section{DISCUSSION}

PrPSc is known to represent the transmissible infectious agent in sheep scrapie, through its ability to promote the structural conversion of the normal cellular prion protein $\mathrm{PrPc}$, into a likeness of itself. Although the existence of a genetic susceptibility for scrapie infection is well known ${ }^{28,29}$, it is reasonable to hypothesize that particular metabolic states in target cells could modulate the degree of susceptibility to prion infection and/or progression to overt disease. In this context, our study on the role of alterations of intracellular cholesterol homeostasis on the pathogenesis of scrapie in sheep, has revealed a positive correlation between an abnormal accumulation of cholesterol esters and the genetic susceptibility to infection.

Of particular relevance, in our view, is the fact that these alterations are found not only in the brain tissue but also in cultured skin fibroblasts from scrapiesusceptible sheep (ARQ/ARQ genotype). As compared to brain tissue, we believe that this in vitro cell system may be amenable to the development of "in vitam" diagnostic laboratory tests. Furthermore skin fibroblasts may help to circumvent possible analytical artefacts due to post-mortem alterations in brain tissue. The molecular mechanisms that cause the increased susceptibility to scrapie in sheep with the ARQ/ARQ genotype are not entirely clear. On the basis of what is known on the familial forms of prion diseases in humans, where mutations in the $P R N P$ gene enhance the rate of spontaneous conversion of PrPc to PrPsc, it is thought that the various polymorphic alleles of $\operatorname{PrP}$ may carry inherently different tendency to acquire PrPsc conformation. Alternatively, however, these polymorphisms could confer increased susceptibility to infection by PrPsc. Our finding that expression of PrP mRNA is increased in sheep with susceptible genotype, may suggest an alternative or complementary mechanism to genetic susceptibility. Indeed, as it has been. proposed to explain prion disease development in PrPc over-expressing mice ${ }^{[37]}$ increased expression of $\mathrm{PrPc}$ could lead to increased production of its altered form (PrPsc).

Recent evidence indicates that the metabolic fate of $\mathrm{PrPc}$ is dependent on its location at the plasma membrane, which in turn is mediated by cholesterol levels in the rafts ${ }^{[11,12]}$, suggesting that the rate of PrPc to PrPsc conversion may be, at least partly, dependent on cholesterol homeostatic regulatory mechanisms. In fact, rafts are essential for the proper folding of nascent $\mathrm{PrPc}$ protein and cholesterol depletion in membranes promotes the conversion of $\mathrm{PrPc}$ to $\mathrm{PrPsc}^{[38]}$. On the other hand, replication of misfolded PrPc protein has been shown to interfere with raft composition, by displacing raft proteins such as Cav-1, leading, among other things, to alterations of intracellular cholesterol trafficking and intracellular cholesterol accumulation $^{[23]}$. We found that cholesterol ester levels were systematically higher in brain tissue and skin 
fibroblasts of scrapie-susceptible sheep (ARQ/ARQ genotype), as compared to those of the scrapie-resistant sheep (ARR/ARR genotype). Accumulation of cholesterol esters was observed in cells from sheep with ARQ/ARQ genotype, whether or not they were infected with scrapie. These data lead to suggest that genetic PrP polymorphisms and alterations of cholesterol homeostasis may act in concert to create an environment favorable to initiation and progression of prion disease.

Further studies are necessary to elucidate the mechanisms responsible for the alterations of cholesterol homeostasis in sheep genetically susceptible to scrapie and to unveil possible cause-effect relationships between $\operatorname{PrP}$ polymorphisms and these alterations. Similarly, additional work is needed to reveal the potential implication of this association for prion disease susceptibility. Nonetheless, the data reported in the present paper suggest that accumulation of cholesterol esters in peripheral cells, together with the altered expression of some proteins implicated in intracellular cholesterol homeostasis, might serve to identify a distinctive lipid metabolic profile associated with increased susceptibility to the prion diseases.

\section{ACKNOWLEDGEMENTS}

The Authors thank Dr. Ciriaco Ligios of Experimental Zooprophylactic Institute of Sassari (Italy) for kindly providing tissue samples from Sarda breed sheep with $\mathrm{ARR} / \mathrm{ARR}$ and $\mathrm{ARQ} / \mathrm{ARQ}$ genotypes

\section{REFERENCES}

1. Thackray, A.M., L. Hopkins and R. Buydoso, 2007. Proteinase K-sensitive disease-associated ovine prion protein revealed by conformationdependent immunoassay. Biochem. J., 15: 475483.

2. Rymer, D.L. and T.A. Good, 2000. The role of prion peptide structure and aggregation in toxicity and membrane binding. J. Neurochem., 75: 25362545.

3. Satoh, J. and Y. Takashi, 2005. Gene expression profile following stable expression of the cellular prion protein. Cell. Mol. Neurob., 24: 793-814.
4. Sorgato, M.C. and A. Bertoli, 2006. Physiopathologic implications of the structural and functional domains of the prion protein. It. J. Biochem., 55: 222-231.

5. Milhavet, O. and S. Lehmann, 2001. Oxidative stress and the prion protein in transmissible spongiform encephalopathies. Brain Res. Rev., 38: 328-339.

6. Liberto, N., S. Prioni, A. Bettiga, V. Chigorno, A. Prinetti and S. Sonnino, 2005. The membrane environment of endogenous cellular prion protein in primary rat cerebellar neurons. J. Neurochem., 95: 771-783.

7. Kurzchalia, T.V. and R.G. Parton, 1999. Membrane microdomains and caveolae. Curr. Op. Cell Biol., 11: 424-431.

8. Simons, K. and R. Ehehalt, 2002. Cholesterol, lipid raft and disease. J. Clin. Invest., 110, 597-603.

9. Diomede, L., G. Forloni, O. Bugiani, F. Tagliavini and M. Salmona, 2002. The prion protein and cellular cholesterol homeostasis. Neurobiol. Lipids, 1: 8-14.

10. Luker, G.D., C.M. Pica, A.S. Kumar, D.F. Covey and D. Piwnica-Worms, 2000. Effects of cholesterol and enantiomeric cholesterol on Pglycoprotein localization and function in lowdensity membrane domains. Biochemistry, 39:7651 -7661 .

11. Prado, A.M., J.A. Silva, Ana C. Magalhaes, V.F. Prado, R. Linden, V.R. Martins and R.R. Brentani, 2004. PrPc on the road: trafficking of the cellular prion protein. J. Neuchem., 88: 769-781.

12. Critchley, P., J. Kazlauskaite, R. Eason and T.J. Pinheiro, 2004. Binding of prion protein to lipid membranes. Biochem Biophys Res Commun., 313: 559-567.

13. Schmitz, G., and E. Orso, 2001. Intracellular Cholesterol and Phospholipid Trafficking: Comparable Mechanisms in Macrophages and Neuronal Cells. Cell Mol. Life Sci., 26:1045-1068

14. Maxfield, F.R., and I. Tabas, 2005. Role of cholesterol and lipid organization in disease. Nature, 438: 612-621

15. Simons, K., and E. Ikonen, 2000. How cell handle cholesterol. Science, 290: 1721-1726. 
16. Feramisco, J.D., A. Radhakrishnamm, Y. Ikeda, J. Reitz, M.S. Brown and J.L. Goldstein, 2005. Intramembrane aspartic acid in SCAP protein governs cholesterol induced conformational change. Proc. Natl. Acad. Sci. USA, 102: 32423247.

17. Brown, M.S. and J.L. Goldstein, 1997. The SREBP pathway: regulation of cholesterol metabolism by proteolysis of a membrane-bound transcription factor. Cell, 89:331-340.

18. Nohturfft A., R.A. Debose-Boyd, S. Scheek, J.L. Goldstein and M.S. Brown, 1999. Sterol regulate cycling of SREBP cleavage-activating protein (SCAP) between endoplasmic reticulum and Golgi. Proc. Natl. Acad. Sci. USA, 96:11235-11240.

19. Binder, W.H., V. Barragan and F.M. Menger. Domains and raft in lipid membrane. Angewandte chemie-international edition, 42: 5802-5827.

20. Shimada A., T. Tamai, K. Oida, S. Takahashi, J. Suzuki, T. Nakai and S. Miyabo, 1994. Increase in neutral cholesterol ester hydrolase activity produced by extralysosomal hydrolysis of highdensity lipoprotein cholesteryl esters in rat hepatoma cells (H-35). Biochim. Biophys. Acta, 1215: 126-132.

21. Oram, J.F. and A.M. Vaughan, 2000. ABCA1mediated transport of cellular cholesterol and phospholipids to HDL apolipoproteins. Curr. Opin. Lipid., 11:253-260.

22. Harris, D.A., 2003. Trafficking, turnover and membrane topology of PrP. Brit. Med. Bull., 66: 71-85.

23. Russelakis-Carneiro, M., C. Hetz, K. Maundrell and C. Soto, 2004. Prion replication alters the distribution of synaptophysin and caveolin 1 in neuronal lipid rafts. Am. J. Pathol., 165:1839-1848.

24. Sarnataro, D., V. Campana, S.M. Paladino, M, Stornaiuolo, L. Nitsch and C. Zurzolo, 2004. PrPC Association with Lipid Rafts in the Early Secretory Pathway Stabilizes Its cellular conformation. Mol. Biol. Cell., 15: 4031-4042.
25. Campana,V., D. Sarnataro, C. Fasano, P. Casanova, S. Paladino and C. Zurzolo, 2006. Detergent-resistant membrane domains but not the proteasome are involved in the misfolding of a PrP mutant retained in the endoplasmic reticulum. J. Cell Sci., 119: 433-442.

26. Collinge, J., 2001. Prion diseases of humans and animals: their causes and molecular basis. Ann. Rev. Neurosci., 24: 519-550.

27. Edskes, H.K. and R.B. Wickner, 2004. Transmissible spongiform encephalopathies: prion proof in progress. Nature, 430: 977-979.

28. Soto, C., and J. Castilla, 2004. The controversial protein-only hypothesis of prion propagation. Nat. Med., 10: S63-67.

29. Wickner, R.B., H.K. Edskes, B.T. Roberts, U. Baxa, M.M. Pierce, E.D. Ross and A. Brachmann, 2004. Prions: proteins as genes and infectious entities. Genes Dev., 18: 470-485.

30. Goldmann, W., M. Baylis, C. Chihota, E. Stevenson and N. Hunter, 2005. Frequencies of PrP gene haplotypes in British sheep flocks and the implications for breeding programmes. J. Appl. Microbiol., 98: 1294-1302.

31. Folch, J., M. Lees and G.H.S. Stanley, 1957. A simple method for the isolation and purification of total lipids from animal tissues. J. Biol. Chem., 226: 497-509.

32. Bowman, R.E. and R.C. Wolf, 1962. A rapid and specific ultramicro method for total serum cholesterol. Clin. Chem., 8:302-309.

33. Lowry, O.H., N.J. Rosebrough, A.L. Farr and R.J. Randall, 1951. Protein measurement with the Folin phenol reagent. J. Biol. Chem., 193: 265-275,

34. Gasparini, L., M. Racchi, G. Binetti, M. Trabucchi, S.B. Solerte, D. Alkon, R. Etcheberrigaray, G. Gibson, J. Blass, R. Paoletti and S. Govoni, 1998. Peripheral markers in testing pathophysiological hypotheses and diagnosing Alzheimer's disease. Faseb J., 12:17-34 
35. Dessì, S., P. La Colla and A. Pani, 2007. Methods for the diagnosis of proliferative and/or conformational diseases. PCT/IT2007/000110.

36. Anchisi, C., S. Dessì, P. La Colla and A. Pani, 2007. Prophylactic and/or therapeutic treatment of proliferative and conformational diseases. PCT/IT2007/000109.
37. Hunter, N., D. Cairns, J.D. Foster, G. Smith, W. Goldmann and K. Donnelly, 1997. Is scrapie solely a genetic disease? Nature, 386:137.

38. Abid K., and C. Soto, 2006. The intriguing prion disorders. Cell. Mol. Life Sci., 63:2342-2351 